\title{
OFERTA E DEMANDA DE TECNOLOGIA: UM ESTUDO DE CASO NO ENTORNO DO PORTO DO AÇU
}

\author{
Fabiane Araújo Leonardo ${ }^{1}$ \\ ORCID: https://orcid.org/0000-0001-5055-718 \\ Henrique Rego Monteiro da Hora ${ }^{2}$ \\ ORCID: https://orcid.org/0000-0001-7192-9245 \\ Edson Terra Azevedo Filho ${ }^{3}$ \\ ORCID: https://orcid.org/0000-0003-4071-5150 \\ Recebido em: 29/03/2021 \\ Publicado em: 30/06/2021
}

\begin{abstract}
RESUMO
O desafio de transformar o trabalho da academia (invenções) em produtos e serviços à disposição da sociedade (inovações) passa pelo relacionamento entre as ICTs e setores produtivos. Contudo, o relacionamento de atores dentro dos ecossistemas não é natural e precisa ser conhecido e incentivado. Este trabalho tem como objetivo analisar a demanda tecnológica da indústria e oferta de inovação pelas ICTs, com estudo de caso com as indústrias instaladas no porto do Açu. O método de localização industrial COPPE-COSENZA foi adaptado para compor matrizes de oferta por parte das ICTs, baseada nas suas competências tecnológicas, e demanda por parte das indústrias, baseadas no perfil das patentes depositadas. Tem-se como resultado uma matriz que apresenta com quais indústrias do porto do Açu uma ICT deveria se relacionar para desenvolvimento tecnológico de competência tecnológica mútua. Observa-se que instituições públicas com sede na territorialidade conseguem se relacionar melhor com a matriz industrial da região.

PALAVRAS-CHAVE: Inovação; COPPE-COSENZA; Porto do Açu; Technology push; Market pull.

\section{TECHNOLOGY SUPPLY AND DEMAND: A CASE STUDY IN THE SURROUNDINGS OF PORTO DO AÇU}

\begin{abstract}
The challenge of transforming the academia work (inventions) into products and services available to society (innovations) involves the relationship between ICTs and productive sectors. However, the players relationship within ecosystems is not natural and requires to be known and encouraged. This work aims to analyze the technological demand of the industry and the offer of innovation by Science and Technology Institutions, with a case study about industries installed in the port of Açu. The COPPE-COSENZA industrial location method was adapted to compose supply matrixes by CTI, based on their technological skills, and demand by industries, based on the patent profile. The result is a matrix that shows which industries in the port of Açu an ICT should relate to for technological development of mutual

\footnotetext{
${ }^{1}$ Universidade Candido Mendes (UCAM). Mestra em Sistemas Aplicados à Engenharia e Gestão pelo Instituto Federal Fluminense (IFF). Email: bia.fabianearaujo@gmail.com.

${ }^{2}$ Instituto Federal de Educação, Ciência e Tecnologia Fluminense (IFF). Doutor em Engenharia de Produção pela Universidade Federal Fluminense (UFF). Email: henrique.dahora@iff.edu.br

${ }^{3}$ Universidade Estadual do Norte Fluminense (UENF). Doutor em Sociologia Política pela Universidade Estadual do Norte Fluminense. Email: etaf@uenf.br.
} 
technological competence. It is observed that public institutions based in territoriality are able to relate better with the industrial matrix of the region.

KEYWORDS: Innovation; COPPE-COSENZA; Port of Açu; Technology push; Market pull.

\section{INTRODUÇÃO}

A atual teoria de mapeamento de tecnologia é tipicamente baseada na inovação de terceira geração dos modelos da década de 1970, combinando modelos lineares de demanda de mercado "market pull", que tentam fornecer os produtos que o mercado exige, e da oferta de tecnologia "technology push", para interessar o mercado em novos produtos desenvolvidos, não abordando as complexas interações em nível sistêmico das organizações (LETABA, 2015; DIXON, 2001).

Os roteiros de tecnologia são apontados como uma metodologia para mostrar direções futuras do mercado e da tecnologia, mas são difíceis de serem feitos pela academia, devido ao objetivo ambíguo do desenvolvimento da tecnologia na universidade, segundo Uchihira (2007). A metodologia proposta por ele reforça o "technology pull" e incentiva a definição primeiro da área de pesquisa, para depois identificar o mercado corresponder.

Ao olhar para demanda de tecnologia, Zocchi \& Tavano (2019) identificaram que $80 \%$ das invenções europeias que são financiadas na área da saúde não são comercializadas, ou seja, não chegam a se tornar inovações. Na indústria farmacêutica da Nigéria, os institutos de pesquisa são mais envolvidos no processo de comercialização de fitoterápicos, que as universidades e, segundo Oseme et al. (2013), o resultado se dá pelo fato de que os institutos são instituições com fins lucrativos e fazem um esforço maior para atender à demanda do mercado, devido à concorrência.

O modelo Tríplice Hélice é frequentemente apontado na literatura e citam a indústria, universidades e as relações governamentais como atores fundamentais no ecossistema. Em uma adaptação, Fain et al.(2010) apresentaram o modelo Hélice Quádrupla, que dá uma atenção especial ao envolvimento do usuário e da sociedade na formação das relações institucionais no processo de inovação.

Considerando que o sucesso de uma invenção se dá no depósito de uma patente e o sucesso da comercialização acontece quando uma patente se transforma em um produto, Dutta \& Hora (2017) identificaram que a incerteza sobre o investimento na comercialização, está relacionada com a escassez de informações sobre as tecnologias inovadoras, com potencial de atender à demanda do mercado. O desafio de desenvolver um planejamento 
estratégico de produtos e tecnologias para apoiar as empresas no processo de inovação, segundo Placzek \& Eberling (2015), está no fato de as informações estarem difusas na web.

Placzek \& Eberling (2015) apresentaram o sistema criado pelo Instituto Heinz Nixdorf, que sincroniza os aspectos da demanda do mercado e oferta de tecnologia. A ferramenta identifica as automaticamente na base de dados do IEEE Xplore as tecnologias, através da mineração de texto. Já Zocchi \& Tavano (2019) propuseram a criação de um Marketplace, que fornece a pesquisadores, inovadores e beneficiários de projetos da União Europeia, oportunidades de parcerias com a indústria e investidores, relacionando todos em um mês ambiente.

O conceito de "technology pull" e "market push" permeiam estudos de caso e metodologias propostas na literatura, mas não há pesquisas profundas sobre encontrar o equilíbrio entre até onde a indústria deve demandar e a academia ofertar. Rothwell (1994) localiza esses relacionamentos como de primeira e segunda geração, e até apresenta uma evolução até a quinta geração. Contudo, relacionamentos maduros, e que geram efetiva inovação não é uma realidade difundida em todas as nações, como não é também na territorialidade onde esse estudo se concentra, estando tanto ainda esta relação nas primeiras gerações.

Deste modo, este trabalho tem como objetivo propor uma adaptação do modelo de localização industrial COPPE-COSENZA, que já foi adaptado para outras aplicações (CHAMOVITZ; DA FONSECA ELIA; COSENZA, 2015), na análise de demanda da indústria e oferta de inovação pelas ICTs (Instituições de Ciência e Tecnologia), com um estudo de caso aplicado ao complexo industrial Porto do Açu e ICTs de Campos dos Goytacazes.

\section{METODOLOGIA}

A seguir serão apresentados a classificação e etapas da pesquisa, a pesquisa metodológica, os procedimentos técnicos e a definição de critérios aplicados neste trabalho.

\subsection{DEFINIÇÃO DOS CRITÉRIOS}

A fim de obter os dados sobre as demandas tecnológicas, o complexo industrial do Açu foi selecionado como base para a pesquisa. Para cada uma das indústrias instaladas no 
parque, os CPCs (Cooperative Patent Classification) foram extraídos dos depósitos de patentes e agrupados para compor os fatores tecnológicos.

O software Orbit da Questel (2020) separa as patentes da empresa selecionada em uma colmeia em uma escala de cores do branco ao vermelho. A intensidade aumenta, à medida que há maior concentração de patentes na área do conhecimento. Dos 35 domínios disponíveis, foram selecionados 32 como fatores tecnológicos, os demais foram desconsiderados por estarem na classificação "outros".

A Matriz A, de demandas tecnológicas, foi classificada da seguinte forma (Quadro 1):

Quadro 1 - Matriz A de demandas tecnológicas

\begin{tabular}{|c|c|l|}
\hline Escala de Cores & Classificação & \multicolumn{1}{|l|}{ Importância de Demanda Tecnológica } \\
\hline & $\mathrm{Cr}$ & Crucial \\
\hline & $\mathrm{C}$ & Condicionante, frequentemente utilizada. \\
\hline LC & Levemente importante. \\
\hline I & Sem demanda encontrada. \\
\hline
\end{tabular}

Fonte: Autores (2020).

A Concentração de patente considerada crucial foi relacionada com as cores abóbora e vermelho e classificada com a sigla "Cr". Já as indústrias das faixas laranja, condicionantes, a demanda tecnológica equivale à classificação " $C$ ". O fator levemente condicionante consiste nas cores bege e amarela, representado pela sigla "LC". E, por fim, foram classificados como inexistentes "I", os fatores tecnológicos que não houve submissão de patente relacionada à empresa.

Na cidade de São João da Barra (RJ), onde está localizado o Complexo Industrial do Porto do Açu não há universidades instaladas. Por se tratar de um polo educacional, foram consideradas as ICTs localizadas na cidade de Campos dos Goytacazes, vizinha à São João da Barra, e centro da mesorregião do Norte Fluminense.

A matriz B, de oferta tecnológica, foi classificada da seguinte forma (Quadro 2):

Quadro 2 - Matriz B de ofertas tecnológicas

\section{Classificação | Critério de Oferta Tecnológica}

Já transferiu tecnologia (patente), executa projeto de extensão

Ex tecnológica na área ou oferece mestrado profissional com relação à competência tecnológica. 


\begin{tabular}{|l|l|}
\hline G & Tem mestrado acadêmico na área do conhecimento. \\
\hline W & Tem graduação ou especialização na área do conhecimento. \\
\hline In & Não há expertise na área. \\
\hline
\end{tabular}

Fonte: Autores (2020).

As ICTs que já transferiram patentes, executam algum projeto de extensão na área do conhecimento ou ofertam mestrado profissional foram classificadas como excelentes "Ex"; as que ofertam mestrado acadêmico na área como boas " $G$ "; as que têm graduação ou pósgraduação como fracas "W"; e quando há inexistência de comprovações, como "In".

O e-Mec (2020) serviu como base para consulta das ICTs localizadas em Campos dos Goytacazes com cursos presenciais de graduação e pós-graduação. Os dados foram confrontados com os sites institucionais e cursos em atividade. Para os cursos de pósgraduação stricto sensu, foi consultado o portal da CAPES (2020). Já as evidências de patentes e projetos de extensão foram realizadas nos sites das ICTs através da busca estruturada no Google (2020), relacionando os termos de pesquisa buscados e levando em conta as objeções (Figura 1).

Figura 1 - Pesquisa estruturada realizada no motor de busca da Google $@$.

site: $\{$ site da ICT $\}$

"Transferência Tecnológica" OR "Transferência de Tecnologia" OR "Parceria com empresa" OR "Parceria industrial" OR "Extensão Tecnológica"

-Estágio -Chamada -Edital

Fonte: Autores (2020).

Além do site da ICT, os termos "transferência tecnológica", "transferência de tecnologia", "parceria com empresa", "parceria industrial" e "extensão tecnológica" foram definidos após várias buscas diretas em notícias e páginas das websites para encontrar termos comuns. O que permitiu também a exclusão dos termos “estágio", "chamada" e "edital”, não considerados como evidências. Além disso, também foram feitas pesquisas nas fundações das ICTs, pois é através delas que as universidades públicas se relacionam com a indústria.

Para execução dosprocedimentos descritos nessa seção, será utilizado o pacote COPPECosenzaR (TARANTI; PESSOA; COSENZA, 2017), disponível no repositório de pacotes do CRAN projeto $\mathrm{R}$. 


\section{RESULTADO E DISCUSSÃO}

Nesta seção serão apresentados os principais resultados encontrados, através da composição da matriz de demanda e da oferta e a matriz de agregação gerada a partir dos fatores tecnológicos estabelecidos.

\subsection{COMPOSIÇÃO DA MATRIZ DE DEMANDA}

Entre as vinte e quatro empresas que estão instaladas ou que mantêm relacionamento de parceria com o Complexo Industrial do Açu. Foram excluídos os parceiros e as empresas que prestam serviço sem relação direta com a atividade fim do Porto. Também foram desconsideradas as Joint ventures, devido ao tempo de criação dessas empresas e a ausência de informações disponíveis. Após a triagem, foram selecionadas onze empresas.

\subsubsection{Anglo American Oil Company}

A Anglo é uma empresa de mineração sul-africana diversificada, focada na extração e processamento de metais do grupo da platina, com operações na África, Europa, América do Sul e do Norte, Austrália e Ásia. Através de uma mina em Minas Gerais conectada ao Porto do Açu, a Anglo American faz parte da Joint Venture da empresa Ferroport, que é responsável pelo terminal de minério de ferro (ANGLO AMERICAN, 2017). A

Figura 2 apresenta os domínios de tecnologia da Anglo American, extraídos das patentes submetidas. 
Figura 2 - Domínios de tecnologias extraídas das patentes submetidas pela Anglo American

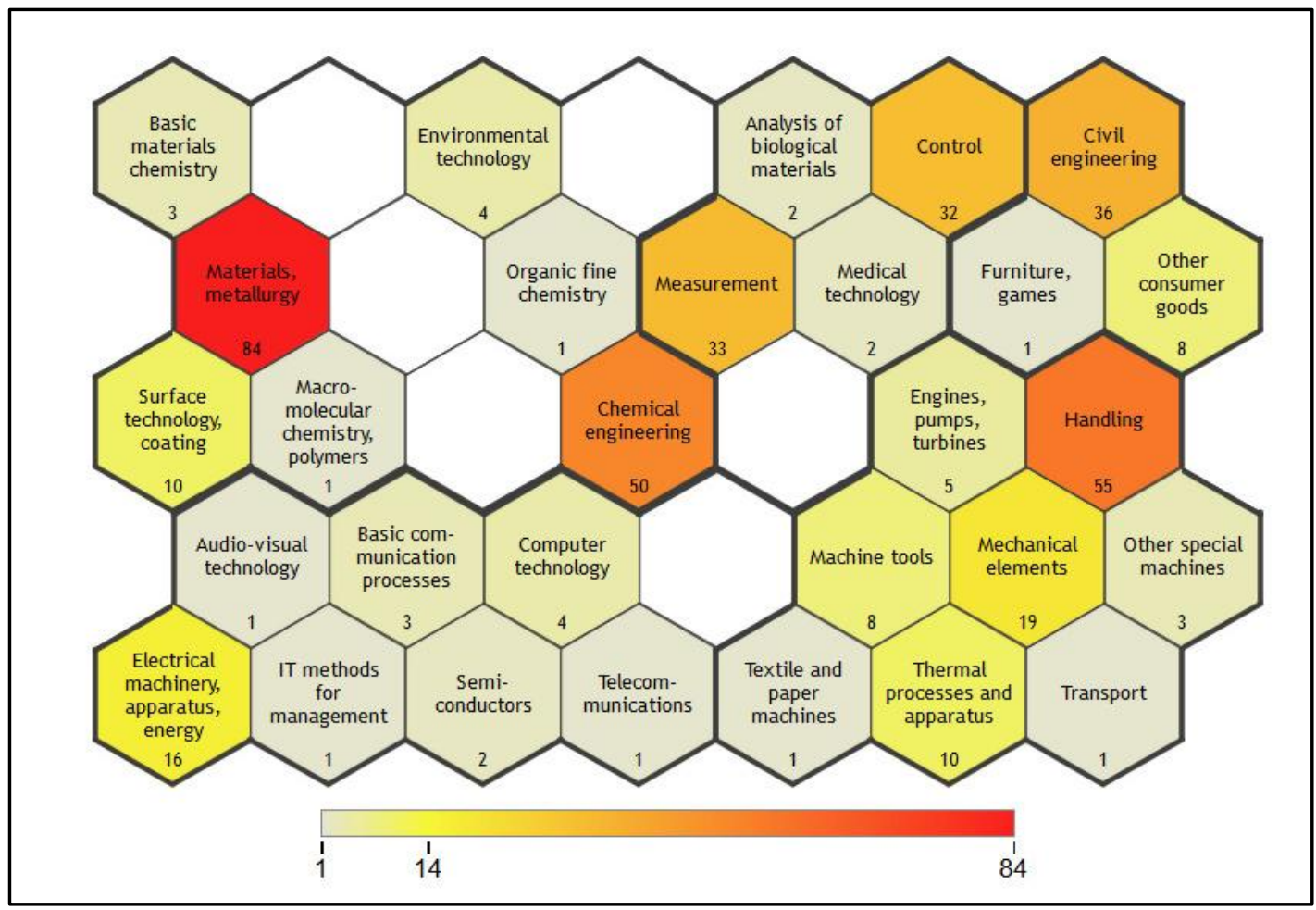

Fonte: Autores (2020).

O fator que apresenta maior concentração patentária está na área de "Materiais e Metalurgia", seguido da "Engenharia Química" e "Manuseio". A empresa tem 84 patentes depositas, com presença em 29 das 35 das áreas.

\subsubsection{Edison Chouest Offshore Inc}

Edison Chouest é uma empresa americana reconhecida como uma das principais fornecedoras de soluções de transporte marítimo do mundo. Sua base no Açu é a maior de apoio à produção de petróleo em alto-mar no mundo, operando com a B-Port. Não foram encontradas patentes relacionadas à organização.

\subsubsection{InterMoor Inc}

A InterMoor é uma empresa integrante do Grupo Acteon e presta serviços de ancoragem para plataformas e FPSO's. A unidade conta com 90 metros de frente de cais e $52.302 \mathrm{~m}^{2}$ de área total. A Figura 3 apresenta os domínios de tecnologia das patentes submetidas pela InterMoor. 
Figura 3 - Domínios de tecnologias extraídas das patentes submetidas pela InterMoor

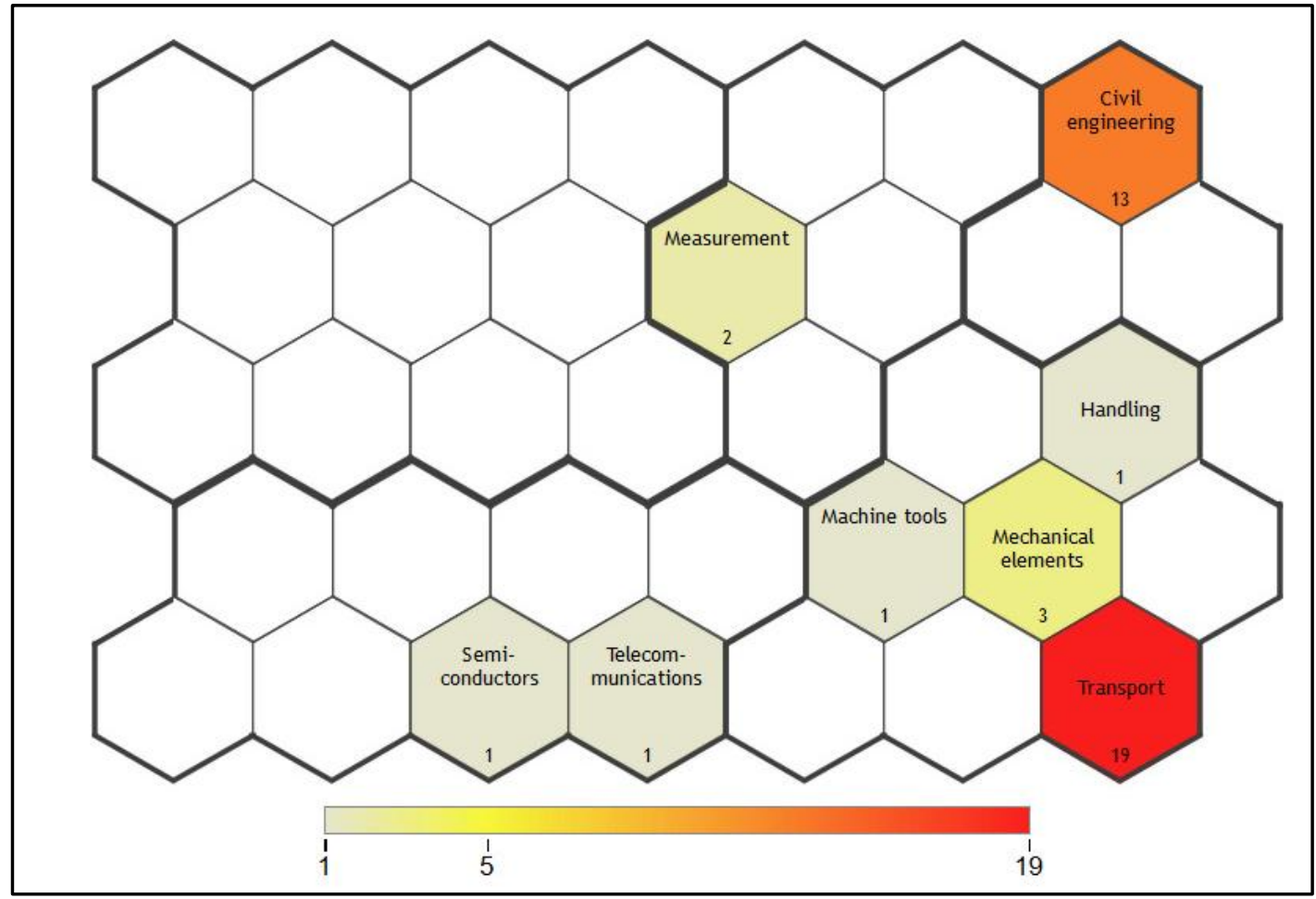

Fonte: Autores (2020).

Ao todo, a Intermoor tem patentes depositadas em oito áreas do conhecimento, tendo maior concentração na área do "transporte", seguida pela "engenharia civil". Totalizando 19 patentes, presentes em 8 dos 35 domínios.

\subsubsection{GranIHC}

Fundada em 2011, a GranIHC é uma empresa que oferece soluções integradas e inovadoras para a indústria com foco na excelência, novas tecnologias e ativos estados da arte. Atua nas áreas de Gerenciamento de Projetos, Serviços de Integridade (IMR), Operações Marítimas e Logística. É sócia da Prumo na DOME, subsidiária criada para prover soluções integradas de logística e serviços para a indústria de O\&G.A Figura 4 apresenta os domínios de tecnologia das patentes da GranIHC. 
Figura 4 - Domínios de tecnologias extraídas das patentes submetidas pela GranIHC

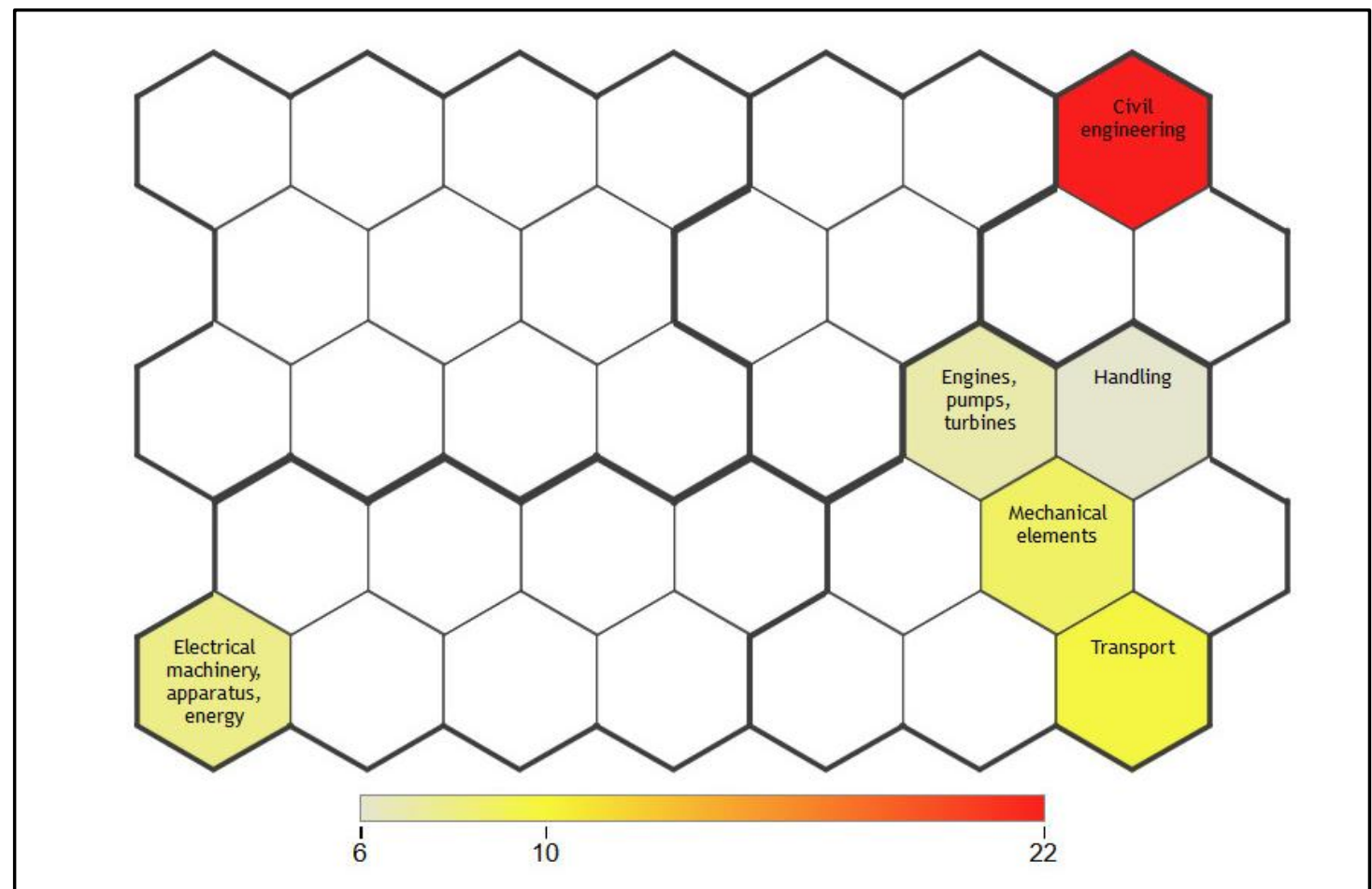

Fonte: Autores (2020).

Entre as empresas selecionadas, que apresentaram patentes depositadas, a GranIHC é a que tem um menor número domínios (17\%) e também o menor número de patentes (22), distribuídos em seis fatores e com forte concentração na "engenharia civil". Podendo significar que a empresa pode utilizar outros mecanismos de proteção do conhecimento, como o segredo industrial, ou as barreiras de entrada são tão grandes, que não há preocupação.

\subsubsection{National Oilwell Varco (NOV)}

A NOV é líder mundial no fornecimento dos principais componentes mecânicos para sondas de perfuração terrestres e marítimas. No porto mantém uma unidade de produção de tubos flexíveis para apoio à indústria offshore. A unidade tem capacidade para produção de $250 \mathrm{~km}$ de tubos flexíveis por ano, além de área para armazenagem e teste de material. A Figura 5 apresenta os domínios de tecnologia da NOV. 
Figura 5 - Domínios de tecnologias extraídas das patentes submetidas pela NOV

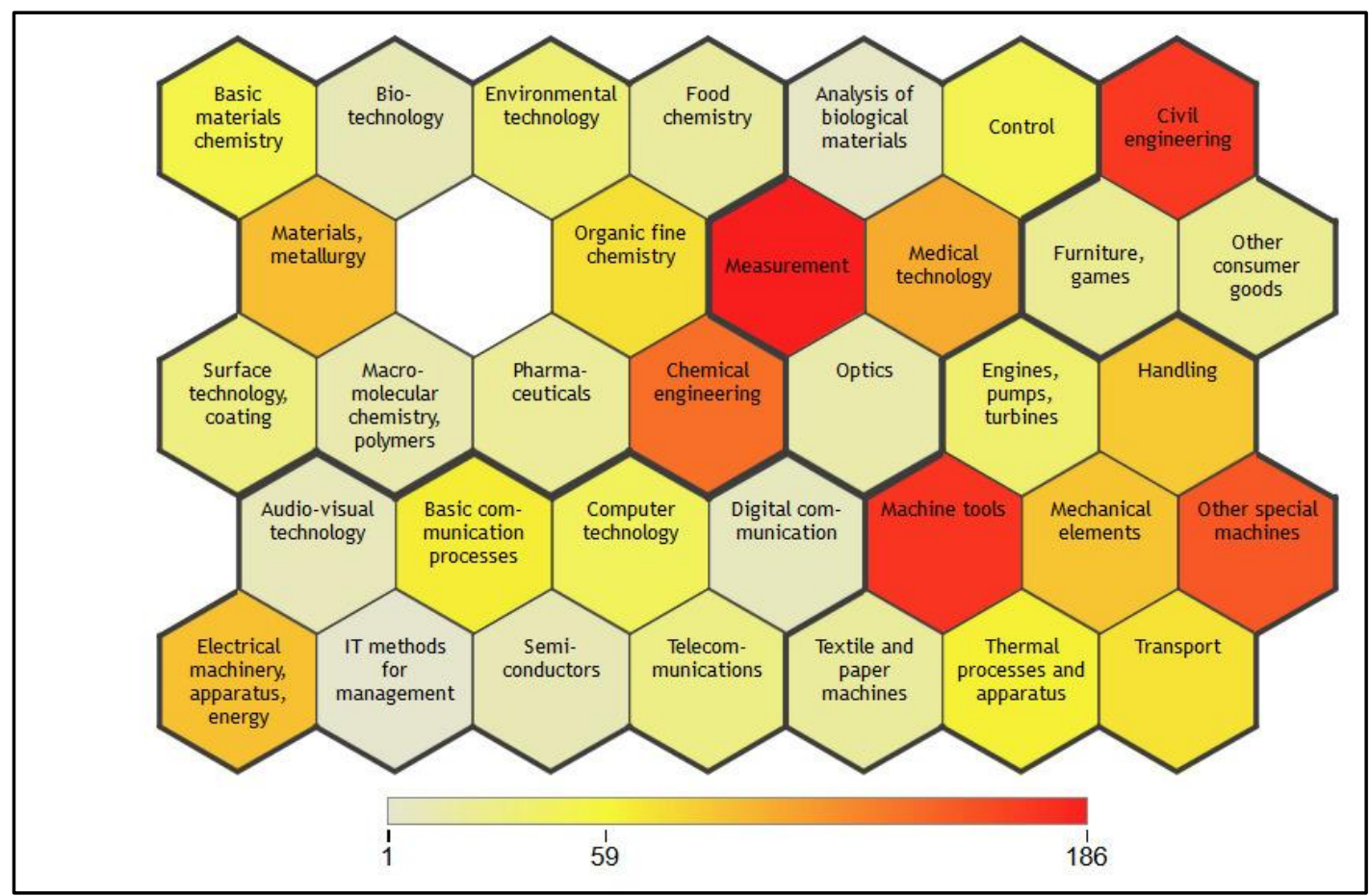

Fonte: Autores (2020).

Com presença em 34 de 35 domínios do conhecimento, a NOV, comparada às demais nesse status, é a que tem menor número de patentes depositadas (186), demonstrando a diversificação da empresa. Também é a única com foco nos domínios de "medição" e "máquinas".

\subsubsection{Petrobrás}

A Petrobras é uma empresa de capital aberto, cujo acionista majoritário é o Governo do Brasil (União). Opera atualmente em 25 países, no segmento de energia, prioritariamente nas áreas de exploração, produção, refino, comercialização e transporte de petróleo, gás natural e seus derivados. Atualmente utiliza seis berços exclusivos na base de apoio offshore da Edison Chouest Offshore. A Figura 6 apresenta os domínios de tecnologia das patentes submetidas pela Petrobras. 
Figura 6 - Domínios de tecnologias extraídas das patentes submetidas pela Petrobras

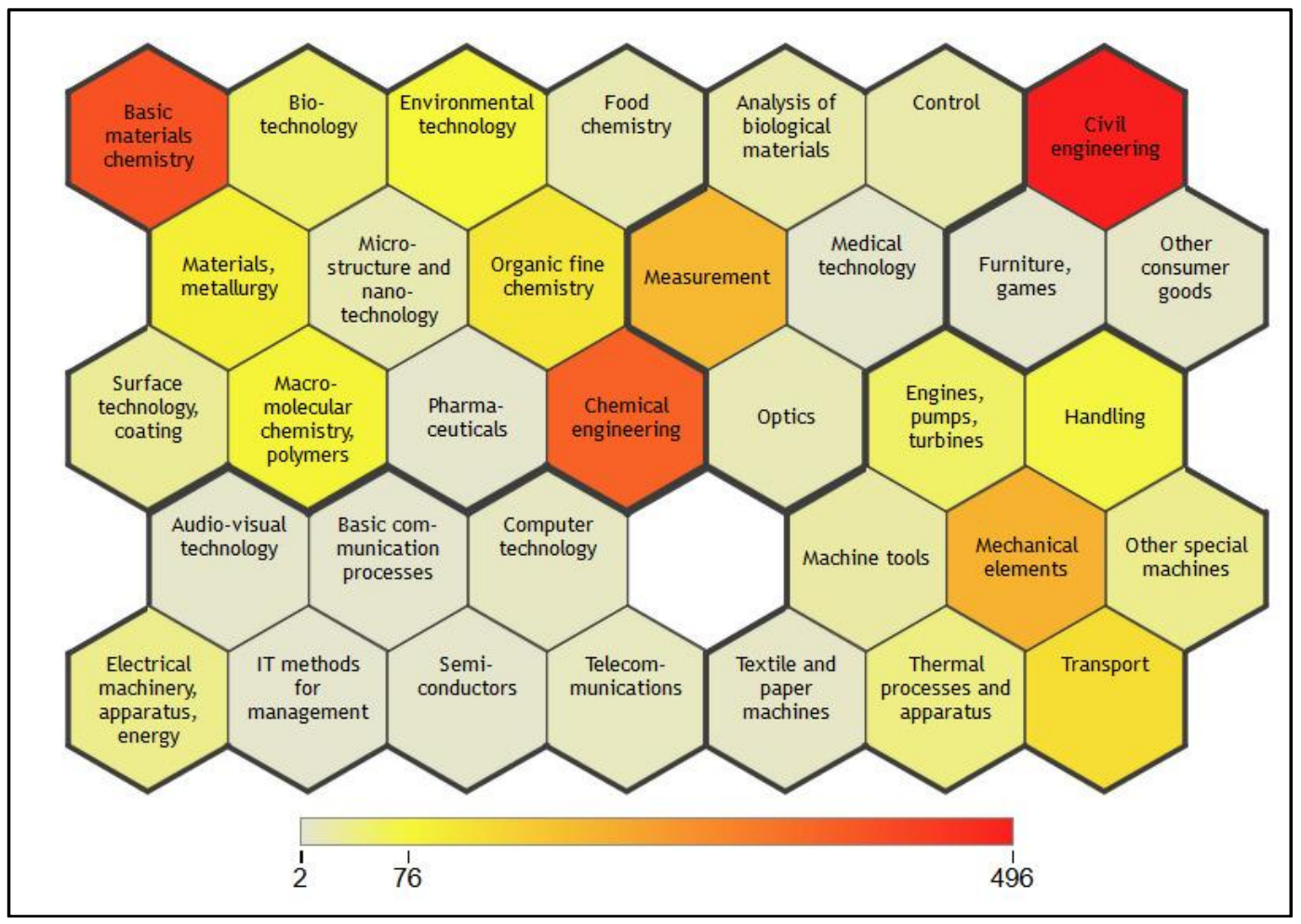

Fonte: Autores (2020).

A Petrobras tem 496 patentes submetidas e está presente em 34 dos 35 domínios do conhecimento, ausente apenas em "Tecnologias Digitais". Chamando atenção ainda para a concentração de patentes na Engenharia Civil, que não corresponde à sua maior atividade fim, que é a geração de energia através do petróleo.

\subsubsection{Royal Dutch Shell (Shell)}

A Shell é uma empresa multinacional petrolífera anglo-holandesa, que tem como principais atividades a refinação de petróleo e a extração de gás natural. Em agosto de 2003, a empresa tornou-se a primeira operadora internacional a produzir petróleo em escala comercial no Brasil, após a abertura do mercado nacional, nos campos de Bijupirá \& Salema. Após a compra da BG em 2016, tornou-se um dos clientes que utilizam os serviços do Terminal de Petróleo (T-OIL) do Porto do Açu. A Figura 7 apresenta os domínios de tecnologias das patentes submetidas pela Shell. 
Figura 7 - Domínios de tecnologias extraídas das patentes submetidas pela Shell

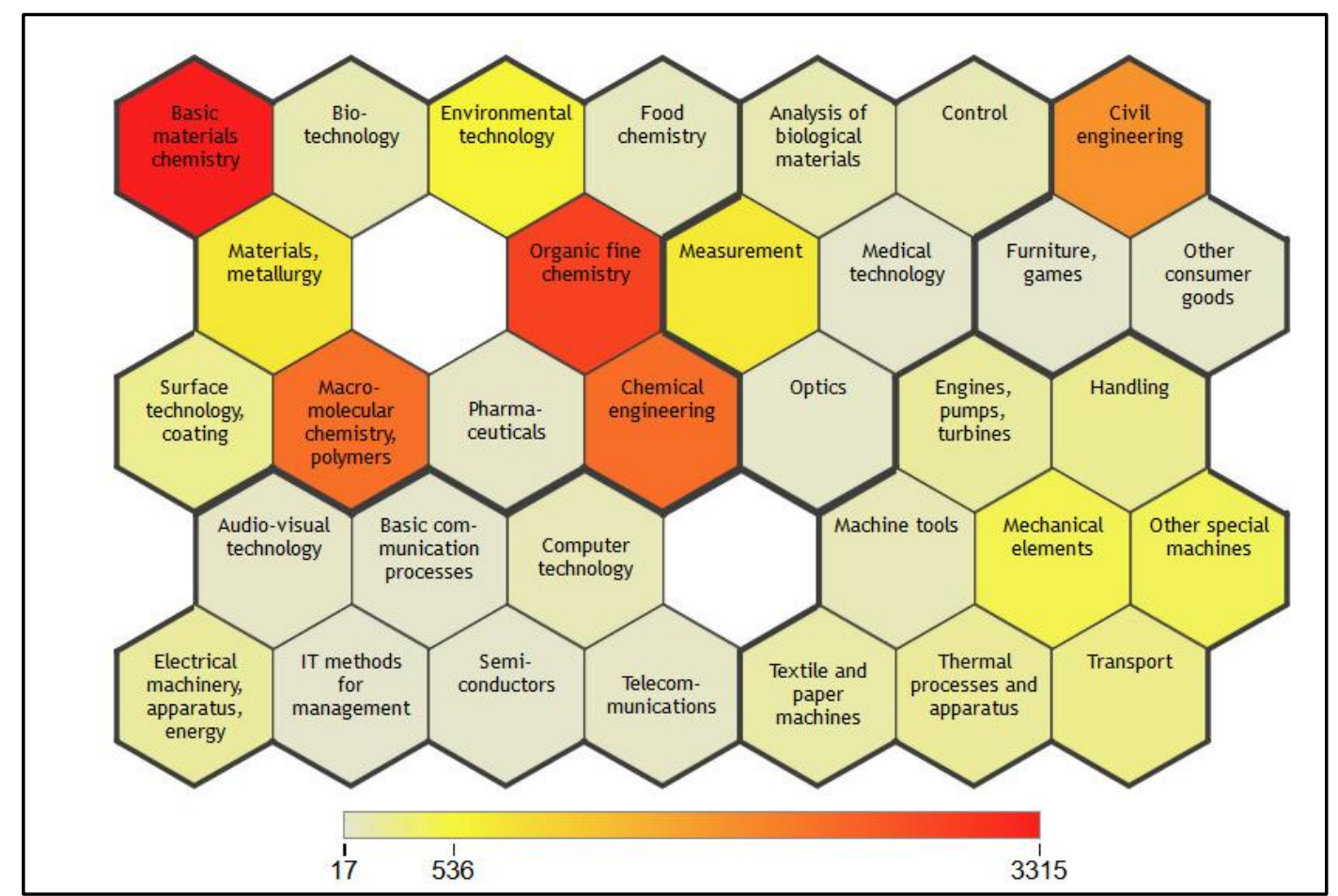

Fonte: Autores (2020).

A Shell trabalha no mesmo segmento que a Petrobras e têm patentes nas mesmas áreas do conhecimento, com maior concentração em "Química" e "Engenharia Civil". Em relação à concorrente, a Shell tem 3315 patentes, contra 496 da Petrobras. Também não tem patentes submetidas em Comunicação Digital" e não apresenta patente em "Microestrutura" e em "Nano Tecnologia", diferente da Petrobrás.

\subsubsection{TechnipFMC}

A francesa Technip e a FMC Technlogies realizaram fusão em 2017, criando a TechnipFMC, líder global nos segmentos subsea, onshoreloffshore e projetos surface. A fábrica de dutos flexíveis do Porto do Açu é a mais moderna do mundo, com o objetivo de expandir o potencial de produção de flexíveis da empresa no país, visando atender, principalmente, às demandas do pré-sal. A Figura 8 - Domínios de tecnologias extraídas das patentes submetidas pela então Technip e a Figura 9 - Domínios de tecnologias extraídas das patentes submetidas pela então FMC Technlogies apresentam os domínios das tecnologias extraídas das patentes pelas empresas Technip e FMC Technlogies, respectivamente. 
Figura 8 - Domínios de tecnologias extraídas das patentes submetidas pela então Technip

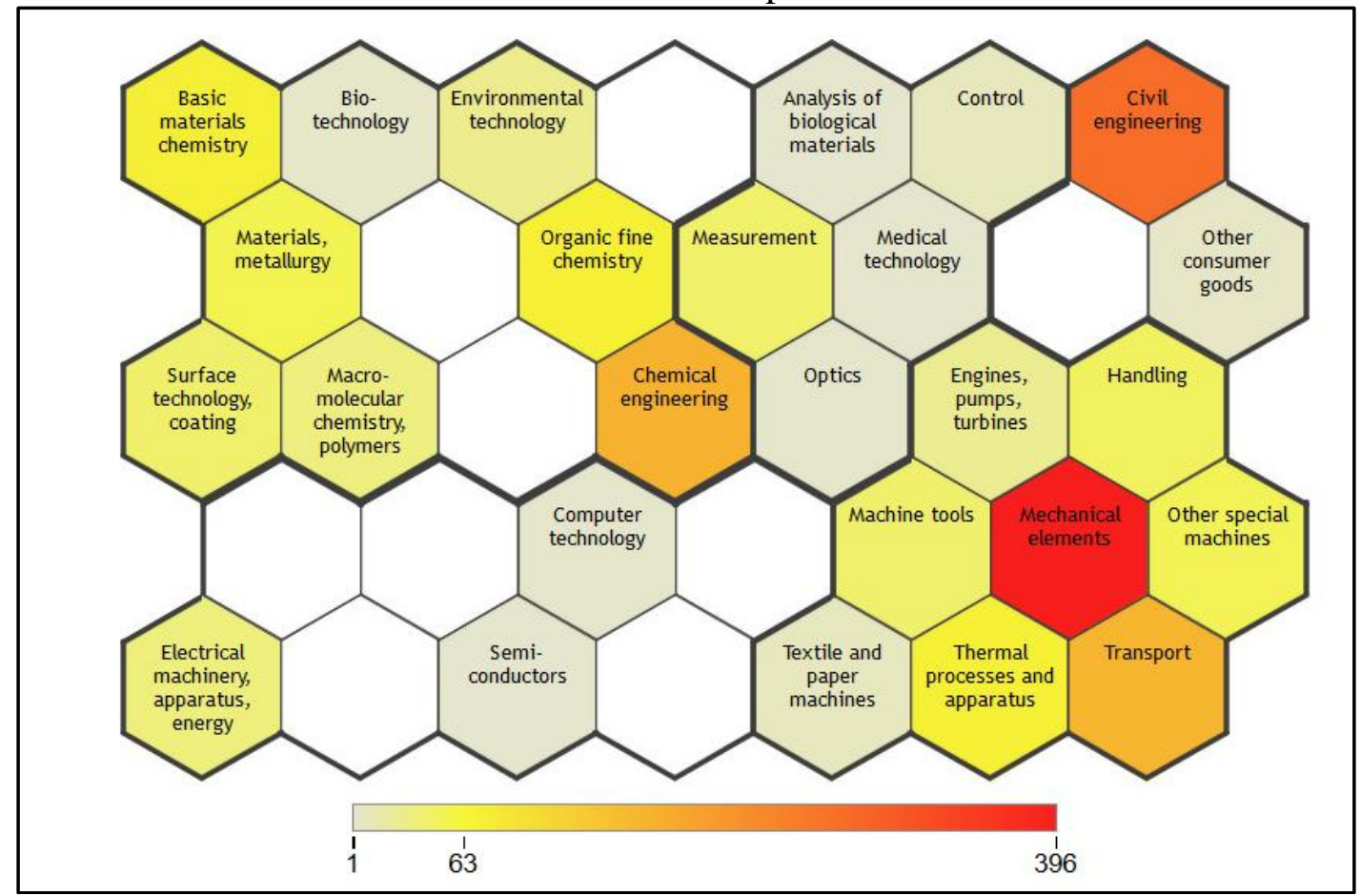

Fonte: Autores (2020).

Figura 9 - Domínios de tecnologias extraídas das patentes submetidas pela então FMC Technlogies

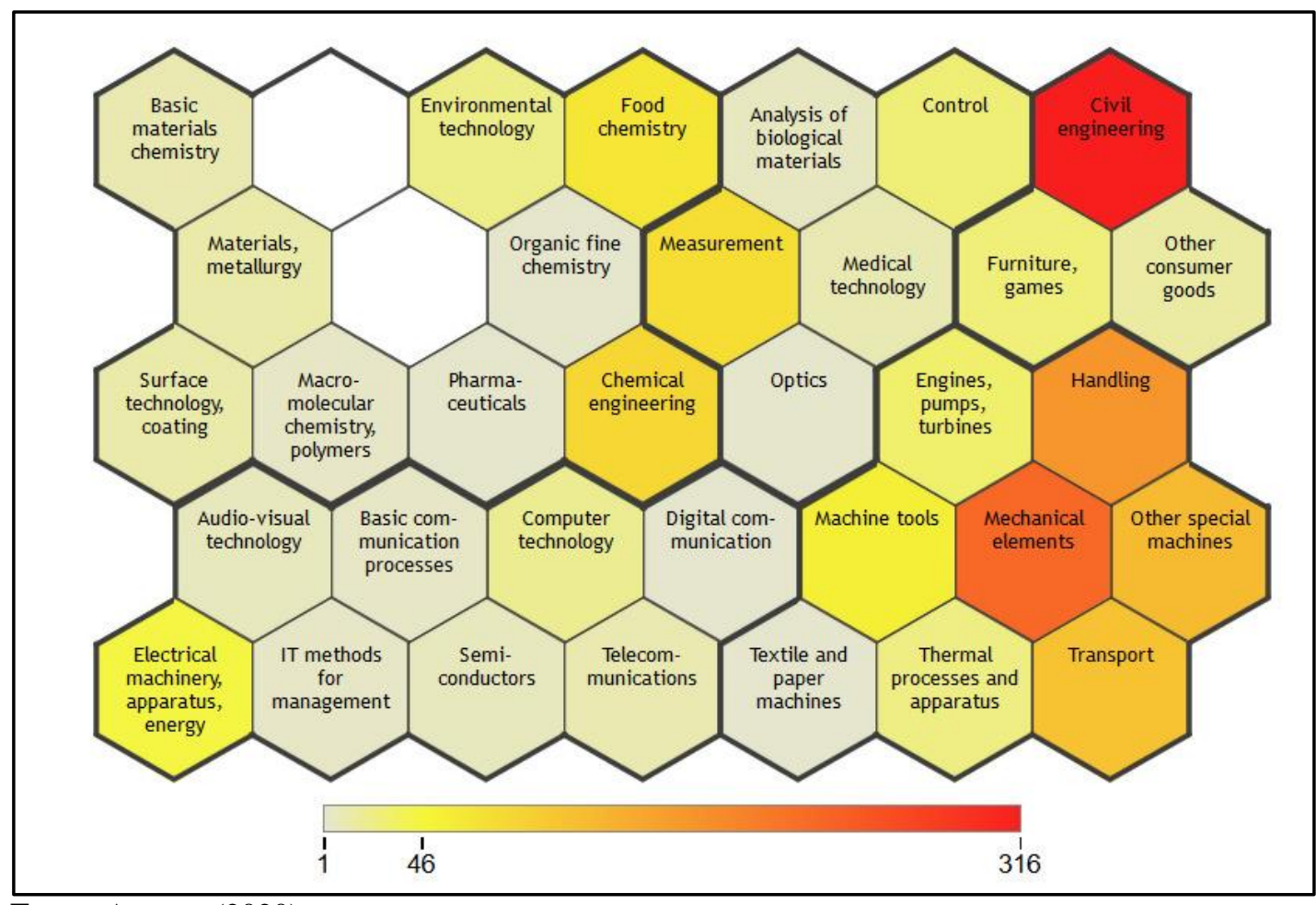

Fonte: Autores (2020). 
Durante uma fusão, as patentes das empresas, também se tornam um patrimônio. Ao analisar as patentes submetidas por ambas as empresas, separadamente foi possível perceber que ambas têm um número muito próximo de submissões - Technip (396) e a FMC (316). Apesar de a FMC ter um número menor de patentes, a cobertura de demandas dela é maior e cobre as demandas da Technip e, por isso, foram consideradas as áreas do conhecimento das patentes submetidas por ela (34 de 35).

\subsubsection{Vallourec}

A Vallourec é líder mundial em soluções tubulares Premium, fornecendo principalmente para o mercado de Energia (Óleo e Gás, Powergen). A base logística no Porto do Açu é destinada ao atendimento das companhias de petróleo que atuam na Bacia de Campos, através da armazenagem e fornecimento "just in time" de tubos e serviços especializados. A Figura 10 apresenta os domínios de tecnologia da Vallourec.

Figura 10 - Domínios de tecnologias extraídas das patentes submetidas pela Vallourec

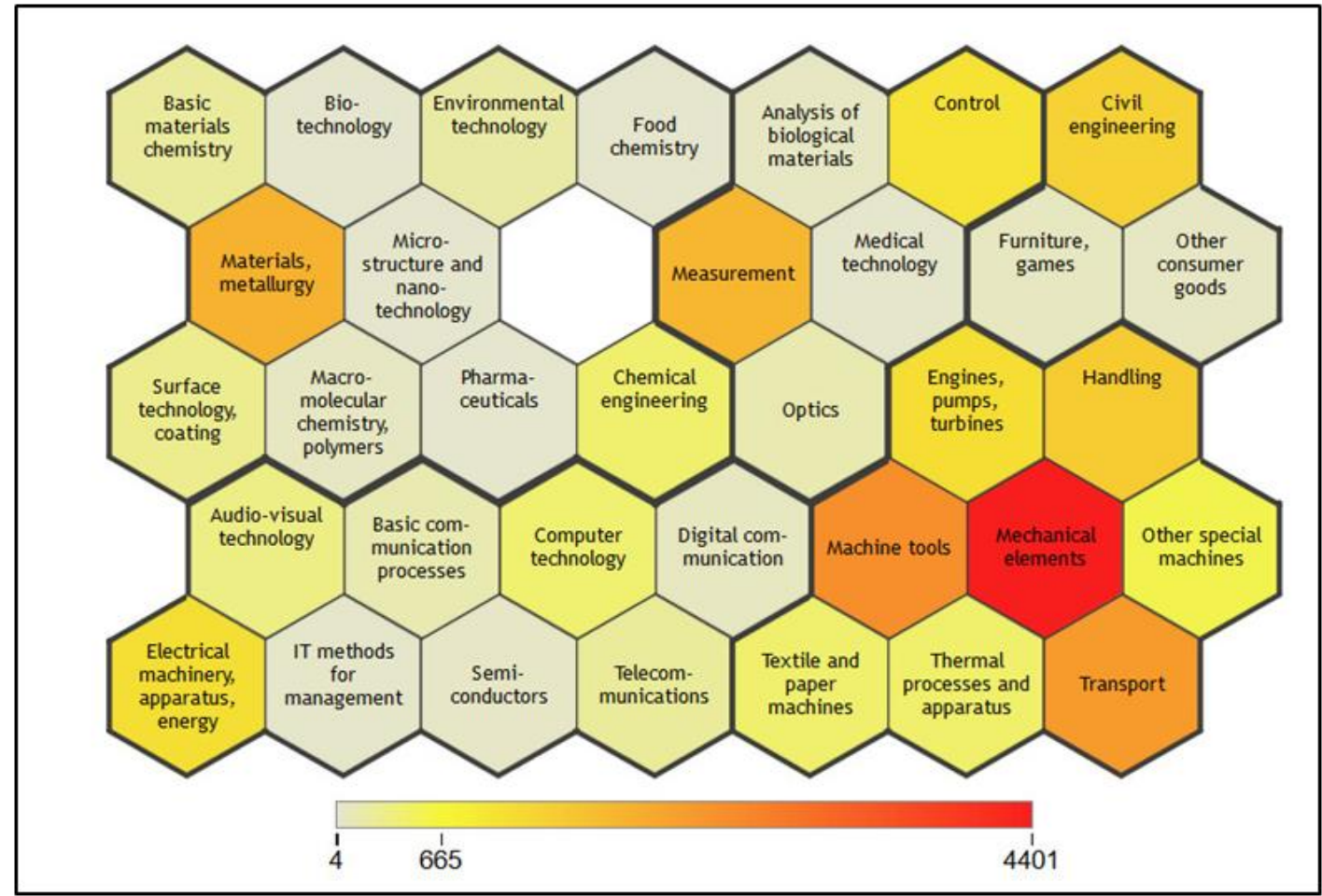

Fonte: Autores (2020). 
Em relação às demais empresas, a Vallourec é a que tem o maior número de patentes (4401). Presente em 34 dos 35 domínios da tecnologia, a Vallourec tem forte concentração na área de Engenharia Mecânica, justificada pela fabricação de soluções tubulares.

\subsubsection{Matriz de Demanda}

Adota-se a postura que uma área de conhecimento é demandada de acordo com a quantidade de investimentos em determinado domínio da tecnologia e, por conseguinte, a quantidade de pedidos de proteção (depósitos) de patentes de invenção em determinado no domínio.

$\mathrm{O}$

Quadro 3 resume a demanda tecnológica das empresas localizadas no Porto do Açu, avaliadas na escala própria para aplicação do método Coppe-Cosenza

Quadro 3 - Matriz de demanda dos fatores tecnológicos pelas empresas do Parque Industrial do Açu

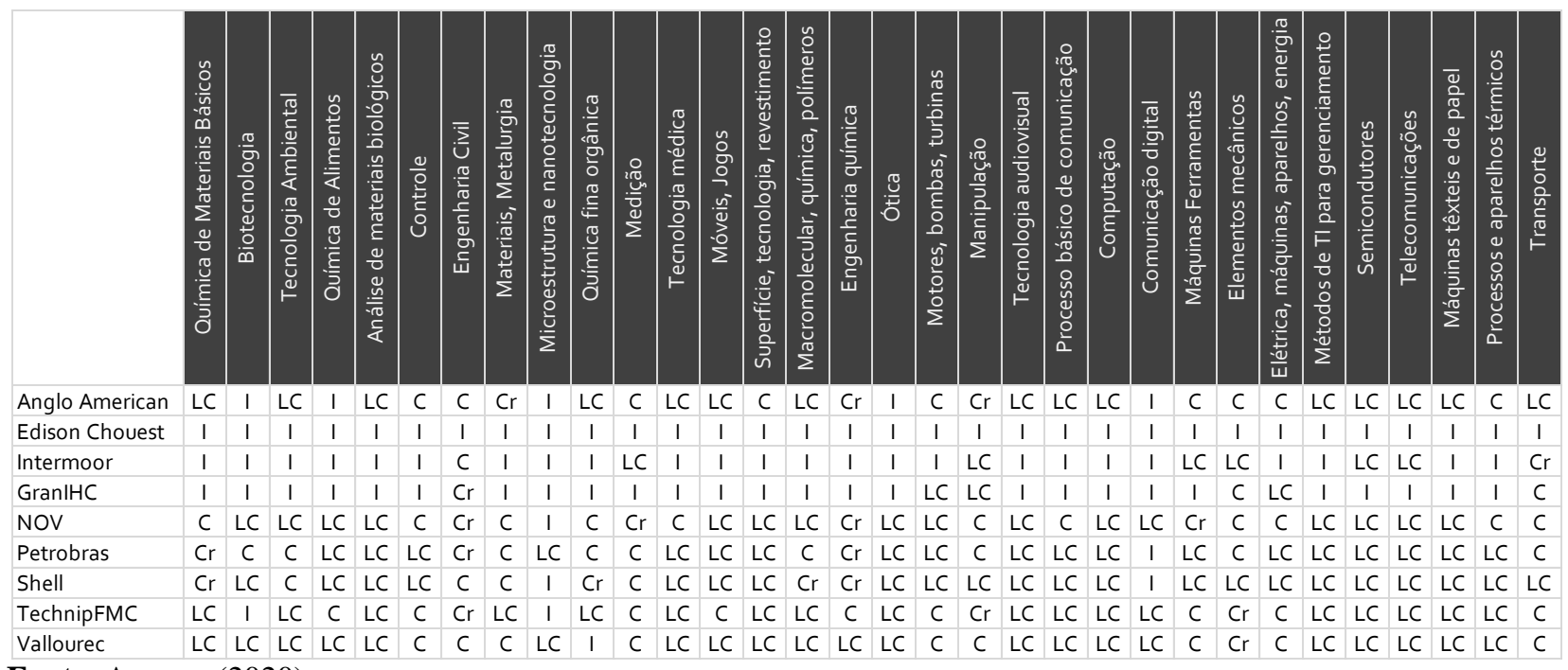

Fonte: Autores (2020).

\subsection{Composição da matriz de oferta}

A seguir, serão descritas as ICTs do entorno do Super porto do Açu, descrevendo as características que sustentem a sua avaliação em cada domínio de tecnologia, à luz do Quadro 4. A fim de selecionar as ICTs, foi realizada uma pesquisa no e-Mec (2020). A partir 
dos resultados encontrados, foi feita uma triagem para identificar instituições com eixo tecnológico. Dessa forma, as instituições que não apresentaram ao menos um curso superior foram desconsideradas, restado dez instituições para análise.

Quadro 4 - Dados sobre a composição das ICTs da matriz de oferta

\begin{tabular}{|c|c|c|c|c|c|c|}
\hline & $\begin{array}{c}\text { Início da operação no } \\
\text { município }\end{array}$ & $\begin{array}{l}\text { Cursos de } \\
\text { Graduaçãa }\end{array}$ & $\begin{array}{c}\text { Cursos de } \\
\text { Especialização }\end{array}$ & $\begin{array}{l}\text { Pós-graduação } \\
\text { acadêmica }\end{array}$ & $\begin{array}{l}\text { Pós-graduação } \\
\text { profissional }\end{array}$ & Categoria \\
\hline IFF & 1909 & 30 & 7 & - & 7 & Pública Federal \\
\hline ISECENSA & 2002 & 10 & 15 & - & - & Privada \\
\hline REDENTOR & 2012 & 9 & - & - & - & Privada \\
\hline UCAM & 1976 & 10 & 7 & 1 & 2 & Privada \\
\hline UENF & 1991 & 17 & 16 & 14 & 1 & Pública Estadual \\
\hline UFF & 1999 & 9 & 3 & 2 & - & Pública Federal \\
\hline UFRRJ & 1991 & - & - & 1 & - & Pública Federal \\
\hline UNESA & 1980 & 12 & 7 & - & - & Privada \\
\hline
\end{tabular}

Fonte: (SILVA NETO et al., 2018).

Foram selecionadas oito instituições, sendo quatro públicas e quatro privadas. Todas as instituições públicas foram consideradas.

\subsubsection{Instituições desconsideradas do estudo}

Por não apresentarem cursos superiores aderentes às áreas do conhecimento da matriz de demanda, as seguintes instituições foram desconsideradas:

- FMC - Faculdade de Medicina de Campos

- FABERJ - Faculdade Batista do Estado do Rio de Janeiro

- UNIFLU - Centro Universitário Fluminense

- UNIVERSO - Universidade Salgado de Oliveira

- FMN - Faculdade Maurício de Nassau

- ISEPAM - Instituto Superior de Educação Professor Aldo Muylaert.

\subsubsection{Matriz de Oferta}

A matriz de oferta foi composta pelas ICTs, seguindo a premissa que são elas as fornecedoras da ciência e tecnologia na região, através do conhecimento produzido e reproduzido, por meio dos cursos de graduação, pós-graduação, mestrado e projetos de pesquisa e extensão: 
Quadro 5 - Matriz de oferta dos fatores tecnológicos pelas Instituições de Ciência e Tecnologia de Campos dos Goytacazes

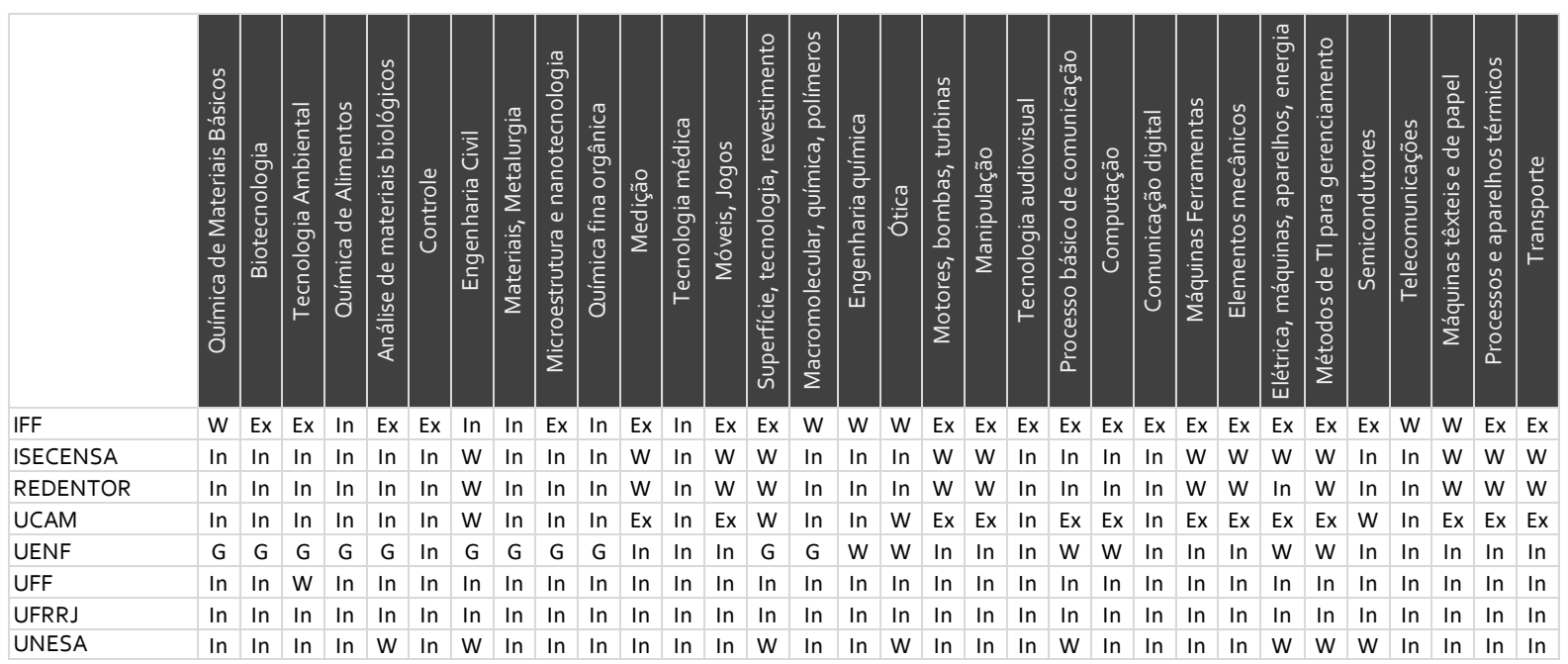

Fonte: Autores (2020).

\subsection{Aplicação do método COPPE-COSENZA}

A aplicação do método considera a demanda dos fatores tecnológicos pelas indústrias, confrontados com a oferta de tecnologia pelas ICTs, resultando na matriz de proximidade entre ambas.

O quadro 6 apresenta o resultado obtido através do cotejo entre as matrizes e a Figura 12

Figura 11 representa os resultados graficamente, a fim de demonstrar de forma visual e facilitada a matriz: quanto maior o círculo, maior o potencial do relacionamento; e, quanto mais escuro, mais pontos de oportunidades de relacionamento.

Quadro 6 - Matriz de Demanda versus Ofertas dos fatores tecnológicos

\begin{tabular}{|c|c|c|c|c|c|c|c|c|}
\hline & IFF & ISECENSA & REDENTOR & UCAM & UENF & UFF & UFRRJ & UNESA \\
\hline Anglo American Oil Company & 23 & 0 & 0 & 14,5625 & 11,34375 & 0 & 0 & 1 \\
\hline Edison Chouest Offshore Inc & 29 & 13 & 12 & 18,21875 & 17,6875 & 1 & 0 & 8 \\
\hline Intermoor Inc & 28 & 7 & 6 & 16 & 17,625 & 1 & 0 & 6 \\
\hline National Oiwell Varco & 22 & 0 & 0 & 13,5 & 10,25 & 0 & 0 & 0 \\
\hline Petrobras & 22 & 0 & 0 & 13,6875 & 9,125 & 0 & 0 & 0 \\
\hline Shell Oil Company & 22 & 0 & 0 & 13,78125 & 8,1875 & 0 & 0 & 0 \\
\hline Fmc Technologies Inc & 22 & 0 & 0 & 13,5 & 10,34375 & 0 & 0 & 0 \\
\hline Vallourec Mannesmann Oil \& Gas & 22 & 0 & 0 & 13,5625 & 11,3125 & 0 & 0 & 0 \\
\hline GranlHC & 28,75 & 7 & 7 & 17 & 15,625 & 1 & 0 & 6 \\
\hline
\end{tabular}

Fonte: Autores. 
Figura 11 - Matriz de Demanda versus Ofertas dos fatores tecnológicos, representada graficamente

\begin{tabular}{|c|c|c|c|c|c|c|c|c|}
\hline & IFF & ISECENSA & \begin{tabular}{|l|l} 
REDENTOR \\
\end{tabular} & UCAM & UENF & UFF & UFRRJ & UNESA \\
\hline Anglo American Oil Company & & & & & & & & $\theta$ \\
\hline Edison Chouest Offshore Inc & & & & & & $\theta$ & & 0 \\
\hline Intermoor Inc & & 0 & 0 & & & $\theta$ & & 0 \\
\hline National Oiwell Varco & & & & & & & & \\
\hline Petrobras SA & & & & & & & & \\
\hline Shell Oil Company & & & & & & & & \\
\hline Fme Technologies Inc & & & & & & & & \\
\hline Vallourec Mannesmann Oil \& Gas & & & & & & & & \\
\hline |HC Engineering Business Limited & & 0 & 0 & & & 0 & & 0 \\
\hline
\end{tabular}

Fonte: Autores.

O IFF apresenta maior aderência às indústrias, não atendendo às demandas das Edison Chouest, Intermoor e IHC. Contudo, o ISECENSA, a REDENTOR, a UCAM, a UENF e a UNESA atendem a esta lacuna. A UCAM e a UENF atendem ainda a todas as demais empresas, com menor intensidade que o IFF.

A UFF e a UFRRJ são universidade públicas com campus em Campos dos Goytacazes, não focados em tecnologia, diferente de suas matrizes. E, devido ao seu posicionamento, não apresentam oportunidade de relacionamento.

O IFF se mostrou mais intenso nas atividades que se propõe a realizar. Já a UCAM e a UENF correspondem à todas as indústrias, mas apresentam mais força para se relacionarem com três empresas.

O ISECENSA, a REDENTOR e a UNESA ainda mantêm atividades muito tímidas, focadas em cursos de graduação e especialização.

Todas as empresas têm potencial para realizar projetos e demandar tecnologias com pelo menos três universidades de Campos dos Goytacazes em diversos níveis. 


\section{CONCLUSÃO}

O objetivo do trabalho foi propor uma adaptação do modelo de localização industrial COPPE-COSENZA, a fim de identificar o potencial de relacionamento entre as ICTs e as empresas localizadas no parque industrial do Açu.

O trabalho teve como base a identificação dos fatores tecnológicos demandados pela indústria através da pesquisa na base de patentes depositadas por cada empresa, identificando a intensidade em cada fator, por meio da distribuição das patentes em cada fator, resultando na matriz de demanda. O resultado foi comparado com a matriz de oferta que, através da avaliação das ICTS, classificada pela oferta de cursos de graduação, especialização, mestrado acadêmico e mestrado profissional, permitiram o preenchimento de cada fator de aderência.

O resultado do cotejo entre a matriz de demanda e a matriz de oferta foi a matriz de demanda versus oferta, que apresentou a capacidade de relacionamento entre as elas.

As instituições públicas com sede no município (IFF e UENF) têm mais potencial para se relacionarem com as indústrias que as que não mantêm a gestão central no território (UFF e UFRRJ). A UCAM, entre as universidades particulares que não mantêm a reitoria na cidade, é a única que detém um poder decisório independente no campus, diferente da UNESA e da REDENTOR. Demonstrando que a gestão da ICT presente no município apresenta um melhor desempenho de entendimento e execução das demandas tecnológicas.

O método utilizou como levantamento das demandas apenas a base de patentes das empresas, devido ao fato de que a maioria delas não publicam com clareza suas demandas e projetos que estão sendo executados. Algumas delas não expõem publicamente seu relatório de gestão, o que gerou um impedimento para utilizar essa fonte na análise. As ICTs por sua vez, não se preocupam em publicar de forma sistemática os projetos e achados de suas pesquisas em seus portais. Corroborando para que não haja clareza entre ambas as partes sobre a demanda e a oferta.

Como proposta de trabalhos futuros, é possível desenvolver canais para integrar a comunicação entre as partes, obtendo fontes externas, que sejam públicas, para automatizar a aplicação do método. 


\section{REFERÊNCIAS}

ANGLO AMERICAN. Relatório à sociedade. Belo Horizonte: Anglo American, 2017. Disponível em: https://brasil.angloamerican.com/ /media/Files/A/Anglo-AmericanGroup/Brazil/imprensa/publicacoes/relatorio-2018-0207a-low.pdf. Acesso em: 11 jun. 2021.

CAPES. PlataformaSucupira. Brasília: UFRN, 2020.

CHAMOVITZ, I.; DA FONSECA ELIA, M.; NUNES COSENZA, C. A. Fuzzy assessment model for operative groups in virtual educational forums. . In: 2015 SCIENCE AND INFORMATION CONFERENCE (SAI). London: IEEE, jul. 2015Disponível em: https://ieeexplore.ieee.org/document/7237173/. Acesso em: 11 jun. 2021.

COSENZA, C. A. N.; NASCIMENTO, P. R. Alguns modelos empíricos de localização industrial. Pesq. Plan. Econ., v. 5, n. 1, p. 261-272, 1975.

DIXON, J. The Market Pull Versus Technology Push Continuum Of Engineering Education. . In: ASEE 2001 ANNUAL CONFERENCE. New Mexico: ASEE, 24 jun. 2001Disponível em: https://peer.asee.org/the-market-pull-versus-technology-pushcontinuum-of-engineering-education. Acesso em: 11 jun. 2021.

DUTTA, D. K.; HORA, M. From Invention Success to Commercialization Success: Technology Ventures and the Benefits of Upstream and Downstream Supply-Chain Alliances. Journal of Small Business Management, v. 55, n. 2, p. 216-235, abr. 2017.

FAIN, N.; MOES, N.; DUHOVNIK, J. The Role of the User and the Society in New Product Development. Journal of Mechanical Engineering, v. 56, n. 8, p. 11, 2010.

GIL, A. C. G. Como elaborar projetos de pesquisa. [s.1.] Atlas, 2010.

LETABA, P.; PRETORIUS, M.; PRETORIUS, L. Technology roadmapping and roadmaps in a context of developing countries: a conceptual framework. Conference Proceedings. Anais... In: INTERNATIONAL ASSOCIATION FOR MANAGEMENT OF TECHNOLOGY INTERNATIONAL ASSOCIATION FOR MANAGEMENT OF TECHNOLOGY. IAMOT, 2015

MINISTÉRIO DA EDUCAÇÃO (MEC). Sistema e-MEC. Brasília: MEC, 2020.

OSEMENE, K. P.; ILORI, M. O.; ELUJOBA, A. Assessing the Commercialization of Herbal Medicines Research and Development outputs in Nigeria. Research Journal of Pharmacy and Technology, v. 6, n. 6, p. 622-631, 28 jun. 2013.

PLACZEK, M.; EBERLING, C.; GAUSEMEIER, J. Conception of a Knowledge Management System for Technologies. 24th International Association for Management of Technology Conference Proceedings. Anais... In: 24TH INTERNATIONAL ASSOCIATION FOR MANAGEMENT OF TECHNOLOGY CONFERENCE. Cape Town: International Association for Management of Technology (IAMOT), IAMOT, 2015Disponível em: http://www.iamot2015.com/2015proceedings/documents/P213.pdf. Acesso em 11 jun. 2021. 
QUESTEL. Orbit Intelligence. França: Questel, 2020.

ROTHWELL, R. Towards the Fifth-generation Innovation Process. International Marketing Review, v. 11, n. 1, p. 7-31, fev. 1994.

SILVA, E. L. S.; MENEZES, E. M. M. Metodologia da Pesquisa e Elaboração de Dissertação. 4. ed. Florianópolis: UFSC, 2005.

SILVA NETO, R. et al. (EDS.). Campos dos Goytacazes Perfil 2018. Campos dos Goytacazes: PMCG, 2018.

TARANTI, P.; PESSOA, L.; COSENZA, C. A. N. coppeCosenzaR. Rio Janeiro: CASNAV, 2017.

UCHIHIRA, N. Future Direction and Roadmap of Concurrent System Technology. IEICE Transactions on Fundamentals of Electronics, Communications and Computer Sciences, v. E90-A, n. 11, p. 2443-2448, 1 nov. 2007.

ZOCCHI, C.; TAVANO, M. D. The EU-Health Innovation Marketplace: facilitating valorization of project results. Materials Today: Proceedings, v. 7, p. 463-469, 2019. 